\title{
Numerical Study of the «Oregonator» Models on the Basis of the Two-Phase Rosenbrock's Method with Complex Coefficients
}

\author{
Svetlana Mustafina, ${ }^{1}$ Rustam Ikramov ${ }^{2}$ \\ ${ }^{1}$ Prof, Dean of Department of Physics and Mathematics, Sterlitamak Branch of the Bashkir State University, \\ Sterlitamak, Russia \\ ${ }^{2}$ Postgraduate student, Department of Physics and Mathematics, Sterlitamak Branch of the Bashkir State University, \\ Russia, Sterlitamak
}

\begin{abstract}
Chemical transformations typically occur according to multiphase schemes. Changes in the concentrations of the starting materials and intermediates with time are not always described with increasing or decreasing functions. A detailed study of a complex process kinetics showed that at the presence of feedback far from equilibrium there may occur vibrational modes - periodic increase or decrease in the concentration of one of the components in time. In a numerical study of oscillating reactions there appears a problem in solving a rigid system of typical differential equations. The purpose of this study is to develop an algorithm and a program to solve the direct kinetic problem and to investigate multi component chemical systems with complex nonlinear dynamics.
\end{abstract}

Keywords: Belousov-Zhabotinsky's reaction, critical reaction, deactivation, Oregonator, oscillations.

\section{INTRODUCTION}

Among the numerous oscillating chemical and biochemical reactions the most famous class of reactions is the class first discovered by the Russian scientists B.P. Belousov and A.M. Zhabotinsky [1]. Belousov-Zhabotinsky's reaction has been studied in hundreds of world laboratories in vessels of various shapes, in a flow, in porous environments, etc.

The mechanism of Belousov-Zhabotinsky's reaction has more than 80 phases. Due to this fact the investigation of the reactions patterns, solutions of the direct and inverse problems as well as the optimization problems are often impossible. In paper [2] there is proposed a simple and abstract model of Belousov-Zhabotinsky's reaction, which turned out to preserve the most important features of this reaction. Such a simplified scheme has been called Oregonator [2].

\section{Data ANALYSeS}

We consider several variants of models of Belousov-Zhabotinsky's oscillatory reaction. We assume that the reaction is carried out in a closed vessel. Then the reaction scheme can be presented as follows [3]:

$$
\begin{aligned}
& A+Y \rightarrow X, \\
& X+Y \rightarrow P, \\
& B+X \rightarrow 2 X+Z, \\
& 2 X \rightarrow Q, \\
& Z \rightarrow f Y,
\end{aligned}
$$

where $A$ and $B$-raw reactants, $P$ and $Q$-products, $X, Y, Z$-intermediates: $\mathrm{HBrO}_{2}, \mathrm{Br}^{-}$and $\mathrm{Ce}(\mathrm{IV})$ correspondingly. Differential equations describing the dynamics of Belousov-Zhabotinsky's reaction (according to a simplified Oregonator scheme) has the following form [4]:

\footnotetext{
${ }^{1}$ Corresponding Author: mustafina_sa@mail.ru
} 


$$
\begin{aligned}
\frac{d X}{d t} & =k_{1} A Y-k_{2} X Y+k_{3} B X-2 k_{4} X^{2}, \\
\frac{d Y}{d t} & =-k_{1} A Y-k_{2} X Y+f k_{5} Z, \\
\frac{d Z}{d t} & =k_{3} B X-k_{5} Z,
\end{aligned}
$$

where $k_{1}=1.34 \mathrm{~mole} / \mathrm{s}, k_{2}=1.6 \cdot 10^{9} \mathrm{~mole} / \mathrm{s}, k_{3}=8 \cdot 10^{3} \mathrm{~mole} / \mathrm{s}, k_{4}=4 \cdot 10^{7} \mathrm{~mole} / \mathrm{s}, A=0.06 \mathrm{~mole}, B=0.06$ mole. Stoichiometric factor $f$ and rate constant $k_{5}$ are parameters related to the consumption of reactants which can be varied [5].

System (2) is characterized by high rigidness coefficient, calculated according to the formula

$$
\mu(t)=\frac{\max \left(\operatorname{Re}\left(-\lambda_{i}\right)\right)}{\min \left(\operatorname{Re}\left(-\lambda_{i}\right)\right)},
$$

where $\lambda_{i}$ - eigenvalues of the Jacobi matrix of the system of differential equations along its solutions and $\operatorname{Re}\left(\lambda_{i}\right)<0$. The rigidness coefficient $\mu(t)$ for system (2) exceeds $1.4 \cdot 10^{6}[6]$.

Thus, for direct kinetic problem (2) explicit schemes for solving typical differential equations become inapplicable. Therefore, the only possible way to solve problem (2) is to use implicit methods.

Let's consider another type of an Oregonator model which takes into account the reaction in an isothermal reactor of a constant volume with metabolism (an open system, ideal mixing reactor). A kinetic scheme with a complex limit cycle will consist of six phases [7]:

$$
\begin{aligned}
& A+X \rightarrow 2 W, \\
& A+Y \rightarrow X+P, \\
& X+Y \rightarrow 2 P, \\
& C+W \leftrightarrow X+Z, \\
& 2 X \rightarrow A+P, \\
& Z \rightarrow C+0.462 Y .
\end{aligned}
$$

This reaction involves 7 substances: $\mathrm{A}=\mathrm{BrO}_{3}^{-}, \mathrm{C}=M(n)$ - ion of a metal catalyst, $\mathrm{P}=\mathrm{HOBr}, \mathrm{W}=\mathrm{BrO}$, $X=\mathrm{HBrO}_{2}, \quad Y=\mathrm{Br}^{-}, Z=M(n+1)-$ an oxidized form of the ion of a metal catalyst. Let's mark the concentration of the reagents in the following way: $c_{1}=\left[\mathrm{BrO}_{3}^{-}\right], c_{2}=\left[\mathrm{Br}^{-}\right], c_{3}=[\mathrm{M}(n)], c_{4}=\left[\mathrm{HBrO}_{2}\right]$, $c_{5}=[\mathrm{HOBr}], c_{6}=\left[\mathrm{BrO}_{2}\right], c_{7}=[\mathrm{M}(n+1)]$. Since reaction (4) takes place in a constant volume isothermal reactor with metabolism, then the corresponding system of differential equations consists of equations of the form:

$c_{1}^{\prime}=-v_{1}-v_{3}+v_{5}+\left(c_{p 1}-c_{1}\right) / \Theta$,

$c_{2}^{\prime}=-v_{1}-v_{2}+0.462 v_{6}+\left(c_{p 2}-c_{2}\right) / \Theta$

$c_{3}^{\prime}=-v_{4}+v_{6}+\left(c_{p 3}-c_{3}\right) / \Theta$

$c_{4}^{\prime}=v_{1}-v_{2}-v_{3}+v_{4}-2 v_{5}+\left(c_{p 4}-c_{4}\right) / \Theta$

$c_{5}^{\prime}=v_{1}+2 v_{2}+v_{5}+\left(c_{p 5}-c_{5}\right) / \Theta$,

$c_{6}^{\prime}=2 v_{3}-v_{4}+\left(c_{p 6}-c_{6}\right) / \Theta$

$c_{7}^{\prime}=v_{4}-v_{6}+\left(c_{p 7}-c_{7}\right) / \Theta$,

Where $\Theta=v / \lambda$ is the time of the mixture in the reactor, $v$ - reactor volume, $\lambda$ - volumetric flow rate of the mixture through the reactor, $v_{i},(i=\overline{1 . .6})$ are given by: 


$$
\begin{aligned}
& v_{1}=k_{1} c_{1} c_{2}-k_{-1} c_{4} c_{5}, \\
& v_{2}=k_{2} c_{2} c_{4}-k_{-2} c_{5}^{2}, \\
& v_{3}=k_{3} c_{1} c_{4}-k_{-3} c_{6}^{2}, \\
& v_{4}=k_{4} c_{3} c_{6}-k_{-4} c_{4} c_{7}, \\
& v_{5}=k_{5} c_{4}^{2}-k_{-5} c_{1} c_{5}, \\
& v_{6}=k_{6} c_{7} .
\end{aligned}
$$

Kinetic constants take the following values: $k_{1}=0.084 \mathrm{~mole} / \mathrm{s}, k_{2}=4 \cdot 10^{8} \mathrm{~mole} / \mathrm{s}, \quad k_{3}=2 \cdot 10^{3} \mathrm{~mole} / \mathrm{s}$, $k_{4}=1.3 \cdot 10^{5} \mathrm{~mole} / \mathrm{s}, \quad k_{5}=4 \cdot 10^{4} \mathrm{~mole} / \mathrm{s}, \quad k_{6}=0.65 \mathrm{~mole} / \mathrm{s}, \quad k_{-1}=10^{4} \mathrm{~mole} / \mathrm{s}, \quad k_{-2}=5 \cdot 10^{-5} \mathrm{~mole} / \mathrm{s}$, $k_{-3}=2 \cdot 10^{7} \mathrm{~mole} / \mathrm{s}, \quad k_{-4}=2.4 \cdot 10^{7} \mathrm{~mole} / \mathrm{s}, \quad k_{-5}=4 \cdot 10^{-11} \mathrm{~mole} / \mathrm{s}$. Initial conditions are given in the form $c(0)=c_{0}$.

After checking the rigidness of the system of differential equations (5), we obtain that $\mu(t)>>4.5 \cdot 10^{5}[8]$.

Thus, for a numerical solution of (2) and (5) it is necessary to develop an algorithm for solving rigid problems with a wide range of sustainability

Due to the large values of stiffness coefficients of systems of ordinary differential equations (2), (5) for a numerical study of Oregonator's models there has been chosen a two-phase Rosenbrock's method with complex coefficients which has L1-stability.

The realization of this method is difficult and requires a large amount of computation. However, this disadvantage is offset by a high resistance, which is an important quality when choosing a method for solving rigid and superrigid systems of differential equations.

The schemes of Rosenbrock's method for a transition to a new time layer require some solutions of a linear system of equations with a well-conditioned matrix which avoids iterations.

Two-phase Rosenbrock's methods general formula can take form in the simplest case[9]:

$$
y_{n+1}=y_{n}+\operatorname{Re}\left(b_{1} g_{1}+b_{2} g_{2}\right) \text {, }
$$

where values $g_{1}$ and $g_{2}$ are obtained from the relevant systems of linear equations with complex numbers:

$$
\begin{aligned}
& {\left[E-h \alpha_{1} f_{y}\left(y_{n}\right)\right] g_{1}=h f\left(y_{n}\right),} \\
& {\left[E-h \alpha_{2} f_{y}\left(y_{n}+h \operatorname{Re}\left(a g_{1}\right)\right)\right] g_{2}=h f\left(y_{n}+h \operatorname{Re}\left(d g_{1}\right)\right) .}
\end{aligned}
$$

Here vector $y_{n}$ - direct numerical solution of the kinetic problem in a time moment $t$, vector $y_{n+1}-$ direct numerical solution of the kinetic problem in the next time step, $h$ - time step, $E$ - identity matrix , $f_{y}$ - Jacobi matrix of system (2) and system (5), values $\alpha_{1}, \alpha_{2}, b_{1}, b_{2}, a$ and $d$-complex parameters defining the properties of the scheme, $\operatorname{Re}(x)$ is real part of complex value $x$. In [9], the following values of the parameters of the method are given:

$$
\begin{aligned}
& \alpha_{1}=0.09705+0.14418 \mathrm{i}, \\
& \alpha_{2}=0.18866+0.06177 \mathrm{i}, \\
& b_{1}=0.04833-0.32059 \mathrm{i}, \\
& b_{2}=0.95166-1.69677 \mathrm{i}, \\
& a=0.53597-0.96659 \mathrm{i}, \\
& d=0.17308-0.16940 \mathrm{i} .
\end{aligned}
$$

The complexity of the algorithm is in the work with complex numbers and matrices of complex numbers. To find vectors $g_{1}$ and $g_{2}$ we have to solve a system of linear algebraic equations with complex numbers. We have to move from complex numbers to real numbers when realizing the algorithm on a computer. To do this, we introduce 


\section{American Research Journal of Mathematics, Volume 1, Issue 1, February 2015}

ISSN 2378-704X

the notation for finding vector $g_{1}$ (vector $g_{2}$ is similar): $A=\left[E-h \alpha_{1} f_{y}\left(y_{n}\right)\right]-\operatorname{complex}$ matrix, $B=h f\left(y_{n}\right)-$ real vector.

First equation (6) can be represented in a matrix form:

$A g_{1}=B$.

Since matrix $A$ and vector $g_{1}$ contain complex numbers, then (7) can be represented as follows: $\left(A_{r e}+i A_{i m}\right)\left(g_{1 r e}+i g_{1 i m}\right)=\left(B_{r e}+i 0\right)$,

where $A_{r e}, g_{1 r e}$ - real part of complex matrix $A$ and vector $g_{1}, A_{i m}, g_{1 i m}$ - corresponding complex parts of matrix $A$ and vector $g_{1}, i$ - an imaginary unit.

To find vector $g_{1}$ it is necessary to solve a system of equations

$$
\left\{\begin{array}{l}
A_{r e} g_{1 r e}-A_{i m} g_{1 i m}=B_{r e}, \\
A_{i m} g_{1 r e}+A_{r e} g_{1 i m}=0 .
\end{array}\right.
$$

Its solution can be written in the following way:

$$
\begin{aligned}
& g_{1 r e}=-A_{i m}^{-1}\left(A_{r e} g_{1 i m}\right), \\
& g_{\text {lim }}=\left(-A_{r e} A_{i m}^{-1} A_{r e}-A_{i m}\right)^{-1} B_{r e} .
\end{aligned}
$$

\section{DISCUSSION}

The integration results of system (2) with initial conditions $X_{0}=5 \cdot 10^{-11}$ mole, $Y_{0}=3 \cdot 10^{-7}$ mole, $Z_{0}=5 \cdot 10^{-8}$ mole, $f=1, k_{5}=0.5$ are presented in Figure 1 - Figure 3. An integration step is $h=10^{-3}$. The system of differential equations (2) is characterized by periodic changes of concentrations with period $T \approx 57.58 c$.

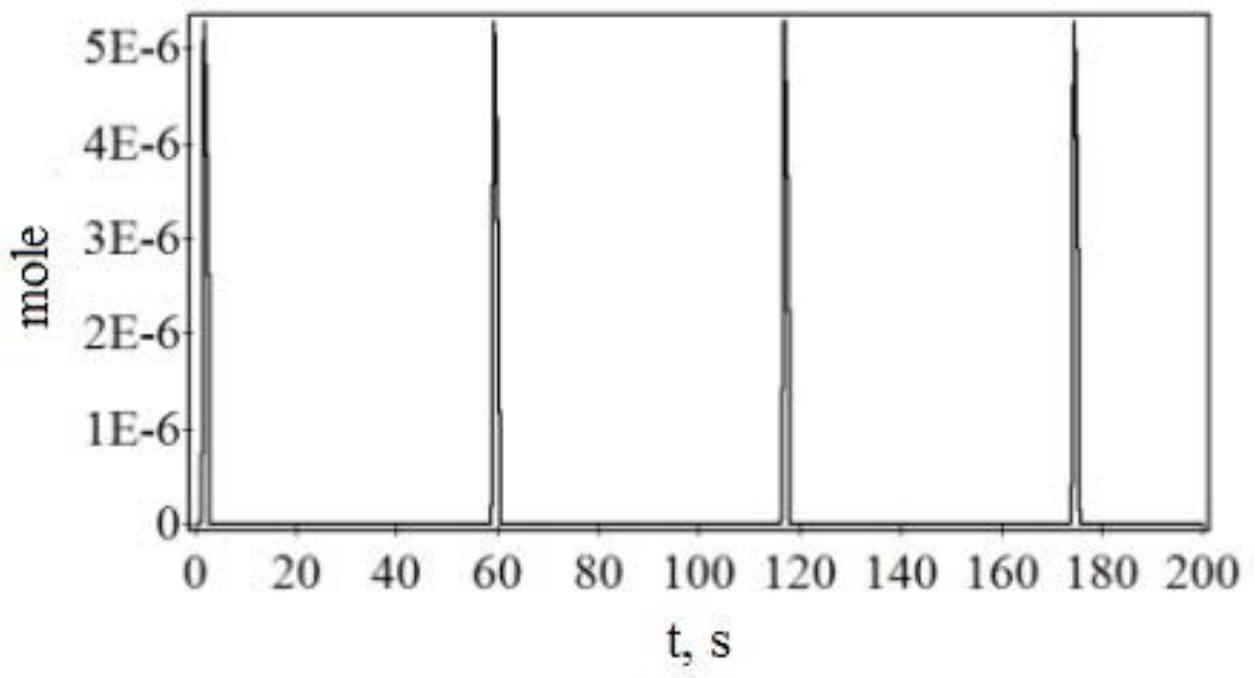

Fig1. Oscillating values of $\mathrm{X}$ reactant concentrations in reaction (2). 


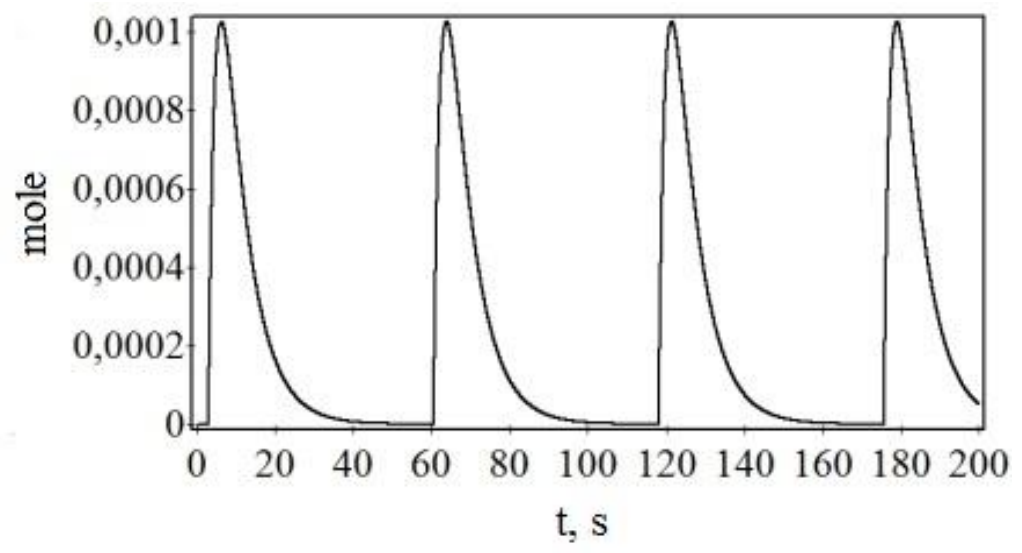

Fig2. Oscillating values of $\mathrm{Y}$ reactant concentrations in reaction (2).

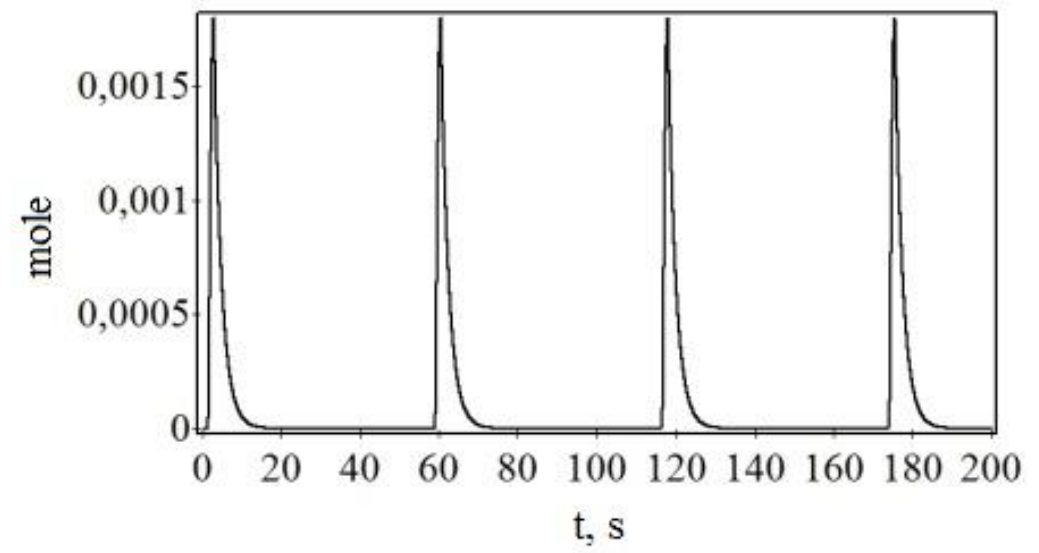

Fig3. Oscillating values of $\mathrm{Z}$ reactant concentrations in reaction (2)

The integration results of system (5) with initial conditions $c_{1}=0.1387$ mole, $c_{2}=1.534 \cdot 10^{-7}$ mole, $c_{3}=1.176 \cdot 10^{-4}$ mole, $c_{4}=3.165 \cdot 10^{-8}$ mole, $c_{5}=1.956 \cdot 10^{-4}$ mole, $c_{6}=5.814 \cdot 10^{-7}$ mole, $c_{7}=6.31 \cdot 10^{-6}$ mole are given in Figure 4 - Figure 5. An integration step is $h=10^{-3}$.

According to the Figures we can see that kinetic curve of reagent $c_{4}$ is characterized by a sinusoidal oscillation mode and kinetic curve of reagent $c_{5}$ by a complex periodic oscillation mode [10].

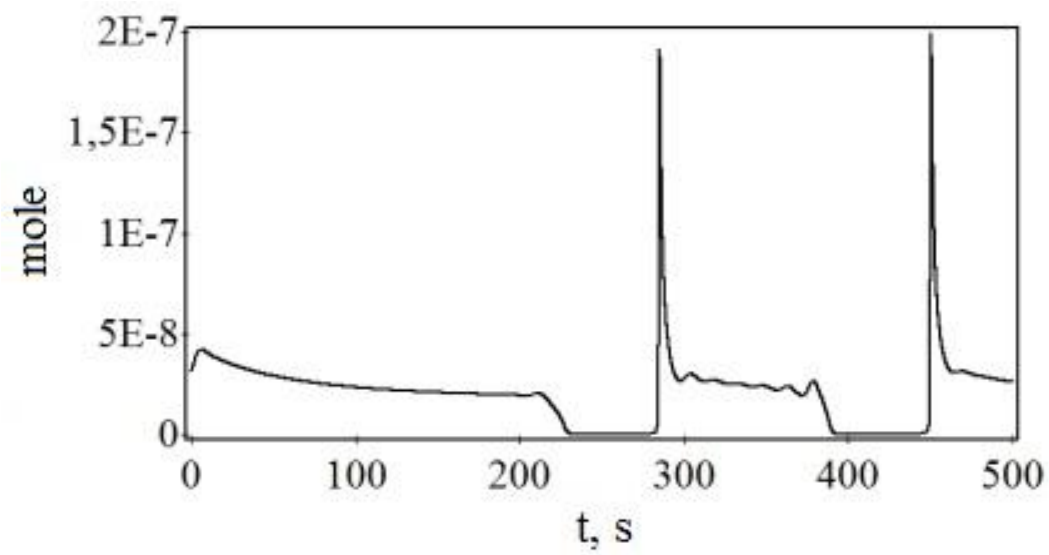

Fig4. Fluctuating values of ${ }^{c_{4}}$ reagent concentration in reaction (5). 


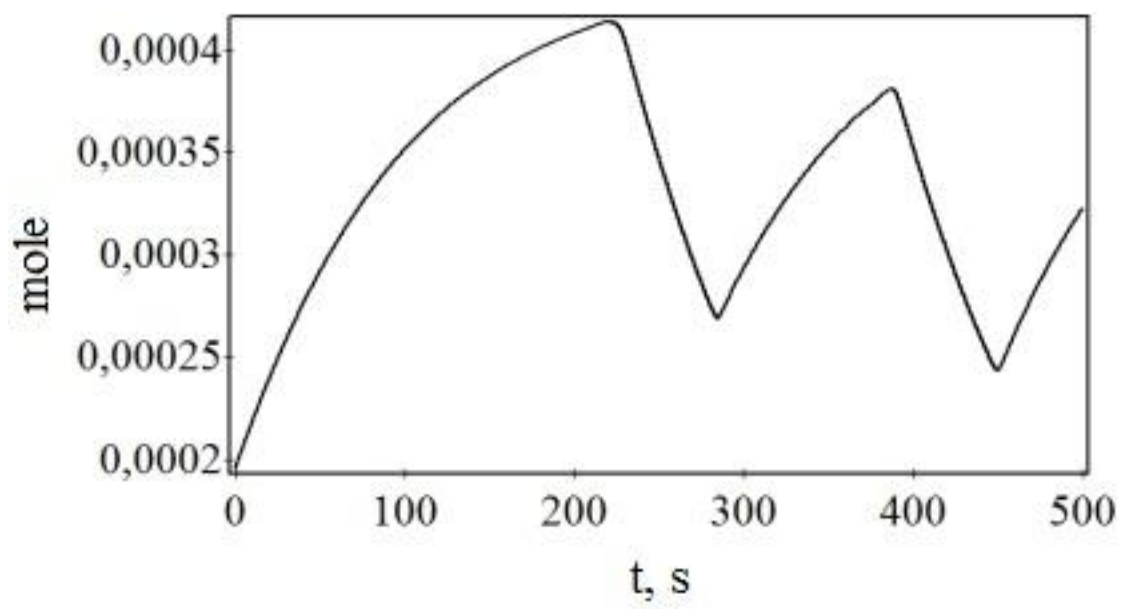

Fig5. Fluctuating values of $c_{5}$ reagent concentration in reaction (5).

\section{CONCLUSION}

The article gives an algorithm for solving the direct kinetic problem based on Rosenbrock's implicit schemes with complex coefficients. An algorithm test has been performed on the famous Belousov-Zhabotinsky's reaction provided with the models taking into account the reactions in a closed and in an isothermal reactor of a constant volume.

A numerical simulation of Belousov-Zhabotinsky's reaction has showed that periodic oscillations of reactant concentrations with period of $T \approx 57,58 c$ can exist in a closed system. Simultaneously, fluctuations in concentrations can be represented by quasi-sinusoidal and complex periodic modes in an isothermal reactor.

There has been worked out a program providing a numerical study of oscillatory reactions in Object Pascal in Lazarus.

\section{REFERENCES}

[1] Belousov, B., 1951, "Periodical reaction and its mechanism", Autowave processes in diffusion systems, p. 76.

[2] Field, R.J. \& Noyes, R.M., 1974, "Oscillations in chemical systems. IV. Limit cycle behavior in a model of a real chemical reaction", Journal of Chemical Physics, 60(5), pp. 1877-1884.

[3] Zhabotinsky A.M., Otmer H., Fild R., 1988, "Kolebanija i begushhie volny v himicheskih sistemah", 720 p.

[4] Vol'kenshtejn M.V., 1988, "Biofizika”, 592 s.

[5] Zhabotinsky, A.M., 1974, "Koncentracionnye avtokolebanija”, 179 p.

[6] Ikramov R., Mustafina S., 2014, "Chislennoe issledovanie modelej oregonatora s ispol"zovaniem dvuhstadijnogo metoda rozenbroka s kompleksnymi kojefficientami“", Informacionnye tehnologii modelirovanija i upravlenija, 3(87), pp 211-217.

[7] Novikov, E.A., 2010, "Numerical modelling of a modified Oregonator with method $(2,1)$ of rigid problem solutions", Computation methods and programming, 11, pp. 281-288.

[8] Ikramov R., Mustafina S., 2014, "Chislennoe issledovanie modelej reakcii belousova-zhabotinskogo na osnove dvuhstadijnogo metoda rozenbroka s kompleksnymi kojefficientami”, Sistemy upravlenija i informacionnye tehnologii, 2(56), pp.11-14.

[9] Limonov, G.A., 2010, “Development of Rosenbrock's two-phase schemes with complex coefficients and their application in the problems of periodic nanostructures modeling", PhD Thesis, Ural Federal Institute, Ekaterinburg, Russia. (Thesis or Dissertation)

[10] Ikramov R., Mustafina S., 2014, "Numerical study of the Belousov-Jabotinsky's reaction models on the basis of the twophase Rozenbrock's method with complex coefficients", International Journal of Applied Engineering Research, 9(22), pp 12797-12801. 